\title{
Integration of robotic surgery into routine practice and impacts on communication, collaboration, and decision making: a realist process evaluation protocol
}

Rebecca Randell ${ }^{1 *}$, Joanne Greenhalgh ${ }^{2}$, Jon Hindmarsh ${ }^{3}$, Dawn Dowding ${ }^{4,5}$, David Jayne ${ }^{6}$, Alan Pearman ${ }^{7}$ Peter Gardner ${ }^{8}$, Julie Croft $^{9}$ and Alwyn Kotze ${ }^{10}$

\begin{abstract}
Background: Robotic surgery offers many potential benefits for patients. While an increasing number of healthcare providers are purchasing surgical robots, there are reports that the technology is failing to be introduced into routine practice. Additionally, in robotic surgery, the surgeon is physically separated from the patient and the rest of the team, with the potential to negatively impact teamwork in the operating theatre. The aim of this study is to ascertain: how and under what circumstances robotic surgery is effectively introduced into routine practice; and how and under what circumstances robotic surgery impacts teamwork, communication and decision making, and subsequent patient outcomes.

Methods and design: We will undertake a process evaluation alongside a randomised controlled trial comparing laparoscopic and robotic surgery for the curative treatment of rectal cancer. Realist evaluation provides an overall framework for the study. The study will be in three phases. In Phase I, grey literature will be reviewed to identify stakeholders' theories concerning how robotic surgery becomes embedded into surgical practice and its impacts. These theories will be refined and added to through interviews conducted across English hospitals that are using robotic surgery for rectal cancer resection with staff at different levels of the organisation, along with a review of documentation associated with the introduction of robotic surgery. In Phase II, a multi-site case study will be conducted across four English hospitals to test and refine the candidate theories. Data will be collected using multiple methods: the structured observation tool OTAS (Observational Teamwork Assessment for Surgery); video recordings of operations; ethnographic observation; and interviews. In Phase III, interviews will be conducted at the four case sites with staff representing a range of surgical disciplines, to assess the extent to which the results of Phase II are generalisable and to refine the resulting theories to reflect the experience of a broader range of surgical disciplines. The study will provide (i) guidance to healthcare organisations on factors likely to facilitate successful implementation and integration of robotic surgery, and (ii) guidance on how to ensure effective communication and teamwork when undertaking robotic surgery.
\end{abstract}

Keywords: Process evaluation, Complex interventions, Realist evaluation, Robotic surgery

\footnotetext{
* Correspondence: r.randell@leeds.ac.uk

'School of Healthcare, Baines Wing, University of Leeds, Leeds LS2 9JT, UK

Full list of author information is available at the end of the article
} 


\section{Background}

The past two decades have seen a revolution in general surgical practice. In the 1990s, traditional open surgery was challenged by the introduction of laparoscopic techniques, initially for benign conditions, but later extended to the treatment of cancer. Instead of producing large abdominal wounds, the surgeon is able to perform operations using small 'key-hole' incisions, through which cameras and instruments are passed. This effectively removes much of the abdominal access trauma. The clinical benefits were soon realised, including less postoperative pain, shorter hospitalisation, quicker return to normal function, and improved cosmetic effect [1-3]. However, laparoscopic operations are technically more challenging than open surgery, as a result of the twodimensional operative image, instrumentation with limited freedom of movement, and lack of tactile feedback. The uptake of laparoscopic surgery has therefore been slow; in 2003, the uptake in colorectal surgery was 5\% and had increased to only $40 \%$ over the 9 years to 2011 [4], despite being recommended by the National Institute for Health and Care Excellence (NICE) since 2006. Robotic surgery offers to solve some of the limitations of the laparoscopic approach. A surgical cart carries four robotic arms, one of which holds the camera, while the other arms hold a variety of surgical instruments. These robotic arms are controlled by the surgeon remotely. The robot provides a stable camera image with threedimensional field of view, with instruments that provide increased freedom of movement, and a digital platform that enables intuitive instrument handling, tremor elimination, and motion scaling. This enables the surgeon to achieve greater precision and control and simplifies many of the tasks that are difficult with traditional laparoscopy.

Enthusiasm for robotic surgery is expressed by both clinicians and policy makers [5-7]. Despite this, the many potential benefits robotic surgery offers for patients are currently not being realised to the full extent because of underuse of surgical robots [8]. Robotic surgery is a complex intervention, by which we mean that it is an intervention aimed at producing change in the delivery and organisation of healthcare services and which comprises a number of separate components that may act both independently and interdependently $[9,10]$. These components are not only technological but also organisational and social, and they can all impact the extent to which the technology is successfully introduced, as well as subsequent process and patient outcomes. The successful performance of a surgical operation is dependent on collaboration amongst staff from different professional groups, including surgeons, anaesthetists, nursing staff, and operating department practitioners (ODPs). There is a complex division of labour that requires the various team members to use their different skills collaboratively to accomplish a single, principal activity [11]. Reports of the use of robotic surgery suggest that a number of factors are important for successful integration, such as having a highly motivated [12] and/or dedicated robotic team [13-15] and additional staff [16]. Operating theatre (OT) staff consider teamwork skills to be critical for easing the integration of robotic surgery, as is having predefined protocols and explicit communication in the event of deviation from the protocol [17]. There is also acknowledgement that there is a learning curve for the whole team [18], not just the surgeon, and that the whole team requires training [19]. However, such recommendations come from small case series (descriptive non-randomised studies) undertaken in single institutions, typically by dedicated robotic surgery enthusiasts [3], so that little is known about the contextual factors that are necessary for the successful integration of robotic surgery into healthcare organisations more broadly.

Existing evaluations of robotic surgery also fail to consider the impact of robotic surgery on communication, teamwork, and decision making in the OT. Robotic surgery significantly changes the spatial configuration in the OT, with the surgeon at a distance from the patient and team. The surgeon's visual attention is focused on the three-dimensional image provided by the robot, inhibiting face-to-face communication during the operative part of the procedure. More generally, the size of the robot introduces physical space constraints, resulting in a new choreography of movement around the patient [17]. The impact of this change in spatial configuration on communication and teamwork in the OT is not a topic that has been explored in evaluations of robotic surgery, which typically focus on the role of the surgeon [20]. The spatial configuration of team members and technology in the OT influence the gathering of information that is used to inform decision making [21,22]. More generally, the spatial configuration of OT teams is not arbitrary but affords particular views of the patient, the rest of the team, and different tools and technologies, with the result that different team members have access to different information to inform their decision making [23]. The nature of the decision making tasks of the OT team may be impacted with robotic surgery. Surgeons report a sense of both physical and psychological isolation from the patient in robotic surgery, and he/she is more dependent on the rest of the team communicating the status of the patient to maintain situation awareness $[17,24]$. Consequently, it has been argued that decision making in robotic surgery is essentially collaborative [24]. In the event of a complication, more of the burden falls on the rest of the team to respond, increasing the importance of the team having 
a shared situation awareness of what is happening in the operative field and how far they are through the procedure [17].

Two small studies have looked specifically at differences in communication between laparoscopic and robotic surgery [25-27]. Both studies found a significant increase in oral communication between the surgeon and the rest of the team in robotic surgery, particularly in relation to the orientation and localisation of organs and the manipulation of instruments, with the effect found to be more pronounced in teams that have less experience in robotic surgery [25]. If use of robotic surgery interferes with standard practices of coordination among the OT team, the achievement of seamless, efficient and timely teamwork may be hampered. It is important to understand any change in communication patterns because of the well-documented relationship between communication and patient safety, with failures in communication and teamwork being identified as key factors in adverse events in the OT [28]. Communication and teamwork around robotic surgery are likely to be influenced by processes associated with the introduction of robotic surgery, such as training and changes in team structure, but equally the integration of robotic surgery in surgical practice may be dependent on the extent to which it is consistent with existing practices for coordination.

For robotic surgery to provide the most benefit for patients, it is first necessary to understand the organisational and social factors that support the successful integration of robotic surgery, by which we mean that it becomes embedded into surgical practice, being used routinely and successfully for surgical operations where it offers advantages to the patient. It is also necessary to understand the impacts of robotic surgery on communication, teamwork, and decision making in the OT and how OT teams manage those impacts.

\section{Aims and objectives}

The aim of this project is to understand how and in what circumstances robotic surgery produces both intended and unintended outcomes. This will be achieved through a realist process evaluation, running alongside an existing randomised controlled trial (RCT) comparing laparoscopic and robotic rectal cancer surgery for the curative treatment of rectal cancer. The study has the following research objectives:

1. To contribute to the interpretation and reporting of the trial results by investigating how variations in implementation of robotic surgery, and the context in which robotic surgery is implemented, impact on outcomes such as operation duration, conversion to open surgery, and complications;
2. To produce actionable guidance for healthcare organisations on factors likely to facilitate successful implementation and integration of robotic surgery; and

3. To produce actionable guidance for OT teams on how to ensure effective communication and teamwork when undertaking robotic surgery.

\section{Methods and design \\ Overall design}

We will undertake a realist process evaluation that will run alongside ROLARR (RObotic versus LAparoscopic Resection for Rectal Cancer), an international, multicentre prospective, randomised, controlled, unblended parallel-group trial, where the primary outcome is conversion to open surgery (as an indicator of technical difficulty) [29]. Process evaluations are predominantly qualitative studies that are typically undertaken alongside a trial, but may be undertaken in preparation for a trial or after a trial [30], and explore how the intervention is implemented [31]. This involves defining the active components of the intervention and investigating contextual factors that affect its implementation.

Realist evaluation is increasingly popular as a method for evaluating the implementation of complex interventions in healthcare [32-36] and has been applied in a variety of fields within health systems research [37]. It does not employ particular methods of data collection but offers a framework for understanding for whom and in what circumstances complex interventions work. It involves building, testing and refining the underlying assumptions or theories of how the intervention is supposed to work [38]. Realist evaluation can complement RCTs in a number of ways [39]. Previously, it has been used in preparation for an RCT, to develop hypotheses that were then tested in the trial $[40,41]$, and for a process evaluation following an RCT, to understand the results of the trial, in terms of how and why the outcomes were achieved $[42,43]$.

\section{Phase I. Formulation of $\mathrm{CMO}$ configurations}

The unit of analysis in realist evaluation is not the intervention but the theories concerning the mechanisms through which the intervention produces certain outcomes in particular contexts. A first task is to identify these theories. In Phase I, the first 'theory elicitation' stage of a realist synthesis will be undertaken to catalogue stakeholders' theories concerning how robotic surgery becomes embedded into surgical practice and its impacts. Such theories are to be found in guidance documentation (e.g., for robotic surgery), position papers, professional journals such as the 'Health Service Journal' and the 'Nursing Times', publications of the Royal Colleges, blogs, thought pieces, advocacy pieces, and critical pieces, and so the review will focus on this grey literature. The output of the review will be a series 
of candidate theories in the form of Context-MechanismOutcome (CMO) configurations.

Telephone interviews will be conducted with staff in National Health Service (NHS) hospital Trusts (providers) in England that are using robotic surgery for rectal cancer resection. This will include Trusts that are participating in the trial and those that are not. Interviews will be semi-structured and conducted using the 'teacher learner cycle' [44]. Here, the interviewer describes, through their interview questions, the candidate theories to the interviewee, who is then invited to comment, expand and discuss the theories based on their experience of the intervention. Through this process, the interviewer channels the interviewee's responses to the task of developing and refining the theories. The interviewer proceeds to formalise the interviewee's theories, based on the information they have given, and the interviewee is then invited to comment on that formalisation. Consequently, the interview is a vehicle for enabling key participants to revise and expand the theory and generate new theories. It is essential that the study captures the perspectives of all professional groups that may influence the effectiveness of the introduction of robotic surgery [24]. Interviews will first be held with one of the surgeons in each Trust and, through them, we will identify other members of the OT team to interview (surgeons, anaesthetists, theatre nurses, ODPs, trainee surgeons) as well as hospital administrators and managers who were involved in the introduction of robotic surgery into the Trust. All interviews will be audio recorded and transcribed verbatim.

An iterative approach to data collection and analysis will be taken in this phase, to support the gathering of further data on emergent themes. Interview transcripts will be entered into a qualitative software programme (NVivo 10) for indexing. Thematic analysis will be used to analyse the data [45]. Following the realist strategy, indexing of the data will focus on identifying interviewees' accounts of how outcome patterns are formed by mechanisms and contexts [43]. In addition, codes developed inductively will be used to index the data. Similarities and differences in the stakeholder theories will be identified and used to further refine the emerging $\mathrm{CMO}$ configurations. Key CMO configurations will then be selected for empirical testing in Phase II.

\section{Phase II. Empirical testing of $\mathrm{CMO}$ configurations}

Four case sites will be selected, three of which will be participants in the ROLARR trial and one which is not (on the basis that only looking at sites in the trial would limit the generalisability of the findings, as sites in the trial could be considered to have successfully embedded robotic surgery into their practice). Case sites will be purposively sampled to ensure variation in the contextual factors identified as being significant in Phase I of the research. These are likely to include: process through which the technology was introduced (organisation led vs. clinician led); level of experience with robotic surgery; and whether dedicated robotic teams are employed.

In realist evaluation, a mixture of qualitative and quantitative methods is important to gather data on the mechanisms and contexts of an intervention as well as its impacts [46]. Data will be collected using a range of methods, which are flexible enough to allow exploration of a variety of CMO configurations. However, the data collection protocol will be revised and further specified at the end of Phase I in light of the theories to be tested.

\section{Data collection}

\section{Structured observation}

Teamwork during operations will be assessed using OTAS (Observational Teamwork Assessment for Surgery) [47]. OTAS comprises ratings on five team behaviour constructs: (i) Communication; (ii) Coordination; (iii) Cooperation and back up behaviour; (iv) Leadership; and (v) Team monitoring and situation awareness. These behaviours are assessed during observation of the surgery, with each behaviour scored on a 7-point scale. OTAS distinguishes between different subteams in the OT (surgeons, anaesthetists, and nurses) and different phases of a procedure (pre-, intra-, and post-operative). For one operation, a total of 45 behavioural ratings are generated (5 behaviour constructs $\times 3$ subteams $\times 3$ phases). Researchers will also record additional information for each operation, such as the team composition, each team member's level of experience of robotic surgery, duration of each phase of the operation, and whether there was a conversion to open surgery.

\section{Video}

To facilitate a close consideration of robotic surgery in use, all operations that are observed will be video recorded. Video recording has been highlighted as an important tool for understanding safety in the OT [48] and has been used successfully in a number of studies concerned with the impact of communication and teamwork on surgical performance [49-51]. The use of two video cameras will allow for the capture of the surgeon's perspective on the surgical scene and the wider conduct of OT team members.

\section{Ethnographic observation}

Ethnography has been argued as an essential approach for studying the introduction of technology into healthcare settings [52]. It is an important complement to the structured observations, allowing unanticipated yet significant behaviours and interactions that fall outside the scope of the observation tool to be recorded in the researcher's field notes. In addition, ethnographic observation is important 
for getting a sense of how what happens in the OT fits within the broader context of work and for capturing those aspects of the context that cannot easily be measured, such as the culture of an organisation. Following in the ethnographic tradition, the researchers will, at least in the early stages of the study, keep the scope of the notes wide on the basis that what previously seemed insignificant may come to take on new meaning in light of subsequent events [53] and should give special attention to the indigenous meanings and concerns of the people studied [54]. In addition, the researchers will record incidents of observer effects (e.g., participants asking 'What are you writing?') to allow analysis of whether participants' awareness of the researchers' presence changed over time [55]. Field notes will be written up as soon after data collection as possible.

\section{Interviews}

Interviews will be semi-structured and will seek to gather data on those outcomes that cannot easily be gathered by other means, particularly those relating to the perceptions of members of the OT team (e.g., level of enthusiasm for robotic surgery, perceptions of teamwork, and perceptions of robotic surgery as an opportunity for training). In addition, due to the infrequency of such events, we are unlikely to see many conversions to open surgery or complications (conversion to open surgery is expected to occur in less than $5 \%$ of operations), so we will gather OT team members' accounts of the reasons for such conversions. All interviews will be audio recorded and transcribed verbatim.

\section{Data analysis}

As in Phase I, an iterative approach to data collection and analysis will be taken. The overall approach to analysis will involve initial comparisons in the processes and outcomes of interest (i.e., those specified in the CMO configurations) between laparoscopic and robotic surgery, before using the data from the robotic surgery operations to test the CMO configurations. We will try to draw on multiple sources of data to test each configuration. Thus, it is through testing the $\mathrm{CMO}$ configurations that the various sources of data will be integrated. Table 1 provides some example theories, based on our current understanding of the literature, and indicates what data could be used for testing each theory.

\section{OTAS}

Initial analysis of OTAS scores will use a mixed model analysis of variance (ANOVA), with case site and sub-team as between-subjects factors and surgery type (laparoscopic vs. robotic), phase, and behaviour as within-subjects factors [47]. We will then test the CMO configurations. For example, this could involve checking for a difference in the overall OTAS score in those case sites where the OT team had been involved in implementation compared to those case sites where they were not involved.

\section{Video}

We will follow standard methods outlined in the literature on video and the analysis of work practice [56], which draw heavily on ethnomethodology [57] and conversation analysis [58]. This analysis will be undertaken by multiple members of the research team, and team 'video review sessions' will be a routine and regular feature of project work. Given the complex and highly specialised character of the setting, specific video extracts and preliminary analytic observations will be discussed with participants at each case site (and the clinical members of the research team) to ensure that the findings are robust, to generate alternative avenues for inquiry and to discuss implications of the findings for practice.

Table 1 Example theories presented as CMO configurations, with data to support testing

\begin{tabular}{|c|c|c|c|c|c|}
\hline Context & + & Mechanism & $=$ & Outcome pattern & Data \\
\hline Experienced teams & + & $\begin{array}{l}\text { Knowledge of how to overcome difficulties in } \\
\text { set-up and positioning of the robot }\end{array}$ & $=$ & $\begin{array}{l}\text { Easier access to patient; Reduced } \\
\text { operation duration; Reduced conversion } \\
\text { to open surgery/complications }\end{array}$ & $\begin{array}{l}\text { Video recordings, } \\
\text { operation duration, } \\
\text { interviews }\end{array}$ \\
\hline $\begin{array}{l}\text { Whole OT team } \\
\text { involved in } \\
\text { implementation }\end{array}$ & + & OT team perceive benefits for patients & $=$ & $\begin{array}{l}\text { Increased motivation among team } \\
\text { members to work together to develop } \\
\text { solutions to problems }\end{array}$ & $\begin{array}{l}\text { Video recordings, } \\
\text { OTAS, interviews }\end{array}$ \\
\hline $\begin{array}{l}\text { Physical separation } \\
\text { between surgeon } \\
\text { and OT team }\end{array}$ & + & OT team less aware of surgeon's actions & $=$ & $\begin{array}{l}\text { Reduced coordination; Increased } \\
\text { operation duration }\end{array}$ & $\begin{array}{l}\text { OTAS, video } \\
\text { recordings, } \\
\text { operation duration }\end{array}$ \\
\hline $\begin{array}{l}\text { Dedicated robotic } \\
\text { teams }\end{array}$ & + & Develop strategies to deal with physical separation & $=$ & $\begin{array}{l}\text { Effective coordination, teamwork, } \\
\text { communication; Reduced operation } \\
\text { duration }\end{array}$ & $\begin{array}{l}\text { OTAS, video } \\
\text { recordings, } \\
\text { operation duration }\end{array}$ \\
\hline $\begin{array}{l}\text { Surgical trainee as } \\
\text { part of team }\end{array}$ & + & $\begin{array}{l}\text { Physical separation and different views of operative } \\
\text { field makes it harder for surgeon to explain what is } \\
\text { happening and monitor trainee's understanding }\end{array}$ & $=$ & $\begin{array}{l}\text { Reduced satisfaction amongst surgeon } \\
\text { and trainee in robotic surgery as } \\
\text { opportunity for training }\end{array}$ & $\begin{array}{l}\text { Video recordings, } \\
\text { interviews }\end{array}$ \\
\hline $\begin{array}{l}\text { More experienced } \\
\text { teams }\end{array}$ & + & $\begin{array}{l}\text { Understand need to support surgeon's situation } \\
\text { awareness }\end{array}$ & $=$ & $\begin{array}{l}\text { Increased verbal communication of } \\
\text { patient state to surgeon; Increased } \\
\text { situation awareness of surgeon }\end{array}$ & $\begin{array}{l}\text { Video recordings, } \\
\text { OTAS }\end{array}$ \\
\hline
\end{tabular}




\section{Ethnographic field notes and interview transcripts}

Field notes and interview transcripts will be entered into NVivo 10 for indexing and will be analysed using the methods outlined for the analysis of the interview data in Phase I. This will identify data to support or refute particular CMO configurations, as well as identifying additional $\mathrm{CMO}$ configurations.

\section{Phase III. Assessing the generalizability of CMO configurations}

Interviews will be conducted at the four case sites with participants from different surgical disciplines to assess the generalisability of the CMO configurations that result from Phase II and to further refine them to reflect the experience of a broader range of surgical disciplines. The interviews will be conducted using the 'teacher learner cycle', as in Phase I. Again, a range of participants (surgeons, anaesthetists, theatre nurses, ODPs, trainee surgeons) from a range of surgical disciplines in which robotic surgery is being used will be included. All interviews will be audio recorded and transcribed verbatim. Interview transcripts will be analysed using the methods outlined for the analysis of the interview data in Phase I.

\section{Ethical considerations}

Phase I of the study has been approved by the University of Leeds School of Healthcare Research Ethics Committee (SHREC/RP/339). NHS Research Ethics Committee approval for Phases II and III of the study has been granted (13/YH/0153).

\section{Discussion}

This paper describes the protocol for a realist process evaluation that uses multiple methods to understand how and under what circumstances robotic surgery is effectively introduced into routine practice; and how and under what circumstances robotic surgery impacts teamwork, communication and decision making, and subsequent patient outcomes. The study will provide actionable guidance for healthcare organisations on factors likely to facilitate successful integration of robotic surgery and for OT teams on how to ensure effective communication and teamwork when undertaking robotic surgery.

There has been much work in recent years on methods for evaluating complex interventions $[9,10,31,59,60]$. At the same time, there has been work on methods for assessing surgical innovations, on the basis of their inherently complex nature $[61,62]$, with some arguing for the inclusion of qualitative data collection alongside RCTs of surgical procedures [63]. Process evaluations are recommended when evaluating complex interventions because, although the RCT design remains as the most reliable method of determining effectiveness [9], it is necessary to understand the mechanisms through which the intervention achieves its outcomes [59]. Without this, effective aspects of the intervention may go unmeasured, raising concerns about the validity and reliability of the results of an evaluation [64] and preventing replication [65]. Process evaluations are particularly important in multicentre trials in which the intervention may be implemented in different ways [31]. Understanding how the components of the intervention and the context vary across sites can assist in interpreting differences in results.

However, the ability of process evaluations to shed light on how an intervention achieves its outcomes has been constrained by a tendency to focus on intervention components rather than on mechanisms through which the outcomes of interest are generated [66]. Where mechanisms are examined, the tendency has been to develop hypotheses about the relationship between intervention components, context, and outcomes, without explicitly testing those hypotheses. The advantage of realist evaluations is that they explain how different contexts trigger particular mechanisms which, in turn, give rise to certain outcomes. Thus it increases the specificity of our understanding of the relationship between context, mechanisms and outcomes.

Discussions with the ROLARR team suggest that the process evaluation will be able to provide insight to support the interpretation of the trial data in terms of understanding any increase in operation duration in robotic surgery and what leads to conversion to open surgery or to complications. In developing the CMO configurations and selecting CMO configurations for testing, attention will be paid to the contexts and mechanisms that may relate to such outcomes. Subject to recruitment, from the data collected in Phase I, we will be able to provide an account of the different ways in which robotic surgery was implemented in all English ROLARR sites, in terms of components of the intervention. Combining these data with the outcome data from the trial will allow for testing of CMO configurations that relate to the trial outcomes and can assist in understanding differences between sites. Inclusion of these findings into the reporting of the ROLARR trial will provide important information for healthcare organisations that are considering introducing robotic surgery.

Despite the successful use of realist methods in process evaluations alongside cluster RCTs of complex interventions and claims by authors of such studies that one of the strengths of realist evaluation is its ability to deal with complexity [37], the role that realist approaches can take in understanding the outcomes of RCTs of complex interventions has been disputed [66-68]. Some would say that realist evaluation is epistemologically antagonistic to the use of RCTs [69], and it has also been claimed that realist evaluation does not deal well with complex multi-site 
interventions with multiple outcomes [69]. Certainly, we would agree with those who have called for further scholarly debate on the place of realist evaluation in RCTs [68], and the proposed study will contribute to that debate, as well as contributing to the development of methods for the evaluation of surgical innovations.

\section{Abbreviations}

CMO: Context-Mechanism-Outcome; NHS: National Health Service; OT: Operating theatre; OTAS: Observational Teamwork Assessment for Surgery; RCT: Randomised controlled trial; ROLARR: RObotic versus LAparoscopic Resection for Rectal Cancer.

\section{Competing interests}

The authors declare that they have no competing interests.

\section{Authors' contributions}

RR is the principal investigator for the study. She conceived, designed, and secured funding for the study in collaboration with JG, JH, DD, DJ, AP, PG, $J C$ and AK. All authors provided input into various aspects of the study design and revised drafts of the protocol. RR led the writing of this protocol manuscript. All authors read and approved the final manuscript

\section{Acknowledgements}

This research is funded by the National Institute for Health Research (NIHR) Health Services and Delivery Research (HS \& DR) Programme (project number 12/5005/04). The views and opinions expressed therein are those of the authors and do not necessarily reflect those of the HS \& DR Programme, $\mathrm{NIHR}$, NHS or the Department of Health.

\section{Author details}

'School of Healthcare, Baines Wing, University of Leeds, Leeds LS2 9JT, UK. ${ }^{2}$ School of Sociology and Social Policy, University of Leeds, Leeds LS2 9UT, UK. ${ }^{3}$ Department of Management, King's College London, London SE1 9NH, UK. ${ }^{4}$ Columbia University School of Nursing, 617 West 168th Street, New York, NY 10032, USA. ${ }^{5}$ Center for Home Care Policy and Research, Visiting Nursing Service of New York, 5 Penn Plaza, New York, NY 10001, USA. 'eeds Institute of Biomedical and Clinical Sciences, University of Leeds, St. James's University Hospital, Leeds LS9 7TF, UK. ${ }^{7}$ Centre for Decision Research, University of Leeds, Leeds LS2 9JT, UK. IInstitute of Psychological Sciences, University of Leeds, Leeds LS2 9JT, UK. ${ }^{9}$ Clinical Trials Research Unit, University of Leeds, Leeds LS2 9JT, UK. ${ }^{10}$ Department of Anaesthesia, Leeds Teaching Hospitals NHS Trust, Leeds General Infirmary, Leeds LS1 3EX, UK.

Received: 7 March 2014 Accepted: 29 April 2014

Published: 2 May 2014

\section{References}

1. Bann S, Khan M, Hernandez J, Munz Y, Moorthy K, Datta V, Rockall T, Darzi A: Robotics in surgery. J Am Coll Surg 2003, 196:784-795.

2. Dobson MW, Geisler D, Fazio V, Remzi F, Hull T, Vogel J: Minimally invasive surgical wound infections: laparoscopic surgery decreases morbidity of surgical site infections and decreases the cost of wound care. Colorectal Dis 2011, 13:811-815.

3. Smith A, Smith J, Jayne DG: Telerobotics: surgery for the 21st century. Surgery (Oxford) 2006, 24:74-78.

4. Lapco National Training Programme for Laparoscopic Colorectal Surgery. [www.lapco.nhs.uk]

5. Mirnezami AH, Mirnezami R, Venkatasubramaniam AK, Chandrakumaran $\mathrm{K}$, Cecil TD, Moran BJ: Robotic colorectal surgery: hype or new hope? A systematic review of robotics in colorectal surgery. Colorectal Dis 2010, 12:1084-1093.

6. NSR Implementation Team: High Quality Care for All: Our Journey So Far. London: Department of Health; 2009.

7. Schreuder HWR, Verheijen RHM: Robotic surgery. BJOG 2009, 116:198-213.

8. Jones A, Sethia K: Robotic surgery. Ann R Coll Surg Engl 2010, 92:5-8.

9. Campbell M, Fitzpatrick R, Haines A, Kinmonth AL, Sandercock P, Spiegelhalter D, Tyrer P: Framework for design and evaluation of complex interventions to improve health. Br Med J 2000, 321:694-696.
10. Medical Research Council: A framework for development and evaluation of RCTS for complex interventions to improve health. In London: Medical Research Council; 2000.

11. Svensson MS, Heath C, Luff P: Instrumental Action: The Timely Exchange of Implements During Surgical Operations. In ECSCW'07: Proceedings of the Tenth European Conference on Computer Supported Cooperative Work; 24-28 September 2007. Edited by Bannon L, Wagner I, Gutwin C, Harper R, Schmidt K. Limerick, Ireland: Springer; 2007:41-60.

12. Goldstraw MA, Patil K, Anderson C, Dasgupta P, Kirby RS: A selected review and personal experience with robotic prostatectomy: implications for adoption of this new technology in the United Kingdom. Prostate Cancer Prostatic Dis 2007, 10:242-249.

13. D'Annibale A, Morpurgo E, Fiscon V, Trevisan P, Sovernigo G, Orsini C, Guidolin D: Robotic and laparoscopic surgery for treatment of colorectal diseases. Dis Colon Rectum 2004, 47:2162-2168.

14. Meehan J, Sandler A: Pediatric robotic surgery: a single-institutional review of the first 100 consecutive cases. Surg Endosc 2008, 22:177-182.

15. Patel VR: Essential elements to the establishment and design of a successful robotic surgery programme. Int J Med Robot 2006, 2:28-35.

16. Rawlings A, Woodland J, Vegunta R, Crawford D: Robotic versus laparoscopic colectomy. Surg Endosc 2007, 21:1701-1708.

17. Lai F, Entin E: Robotic surgery and the operating room team. Proc Hum Factors Ergon Soc Annu Meet 2005, 49:1070-1073.

18. Jayaraman S, Davies W, Schlachta C: Getting started with robotics in general surgery with cholecystectomy: the Canadian experience. Can J Surg 2009, 52:374.

19. Herron D, Marohn M: Group TS-MRSC: a consensus document on robotic surgery. Surg Endosc 2008, 22:313-325.

20. Sgarbura O, Vasilescu C: The decisive role of the patient-side surgeon in robotic surgery. Surg Endosc 2010, 24:3149-3155.

21. Johnson R, O'Hara K, Sellen A, Cousins C, Criminisi A: Exploring the potential for touchless interaction in image-guided interventional radiology. In CHI 2011. Vancouver, BC: ACM; 2011.

22. Mentis H, O'Hara K, Sellen A, Trivedi R: Interaction Proxemics and image use in neurosurgery. In CHI 2012. Austin, Texas: ACM; 2012.

23. Goodwin D: Upsetting the Order of Teamwork: Is 'the same way every time' a good aspiration? Sociology 2007, 41:259-275.

24. Healey A, Benn J: Teamwork enables remote surgical control and a new model for a surgical system emerges. Cogn Technol Work 2009, 11:255-265.

25. Nyssen A-S, Blavier A: Verbal Communication as a Sign Of Adaptation In Socio-Technical Systems: The Case Of Robotic Surgery. In Proceedings of NDM9, the 9th International Conference on Naturalistic Decision Making; June 2009. London: British Computer Society; 2009:267-272.

26. Webster JL, Cao CGL: Lowering communication barriers in operating room technology. Hum Factors 2006, 48:747-758.

27. Cao CGL, Taylor H: Effects of New Technology on the Operating Room Team. In Work with Computing Systems. Edited by Khalid HM, Helander MG, Yeo AW. Kuala Lumpur: Damai Sciences; 2004.

28. Hull L, Arora S, Aggarwal R, Darzi A, Vincent C, Sevdalis N: The impact of nontechnical skills on technical performance in surgery: a systematic review. J Am Coll Surg 2012, 214:214-230.

29. Collinson F, Jayne D, Pigazzi A, Tsang C, Barrie J, Edlin R, Garbett C, Guillou P, Holloway I, Howard H, Marshall H, McCabe C, Pavitt S, Quirke P, Rivers C, Brown J: An international, multicentre, prospective, randomised, controlled, unblinded, parallel-group trial of robotic-assisted versus standard laparoscopic surgery for the curative treatment of rectal cancer. Int J Colorectal Dis 2012, 27:233-241.

30. Lewin S, Glenton C, Oxman AD: Use of qualitative methods alongside randomised controlled trials of complex healthcare interventions: methodological study. Br Med J 2009, 339:b3496.

31. Oakley A, Strange V, Bonell C, Allen E, Stephenson J: Process evaluation in randomised controlled trials of complex interventions. Br Med J 2006, 332:413-416.

32. Goicolea I, Vives-Cases C, Sebastian MS, Marchal B, Kegels G, Hurtig A-K: How do primary health care teams learn to integrate intimate partner violence (IPV) management? A realist evaluation protocol. Implement SC 2013, 8:36.

33. Ranmuthugala G, Cunningham FC, Plumb JJ, Long J, Georgiou A Westbrook Jl, Braithwaite J: A realist evaluation of the role of communities of practice in changing healthcare practice. Implement $S c i$ 2011, 6:49. 
34. Rycroft-Malone J, Fontenla M, Bick D, Seers K: A realistic evaluation: the case of protocol-based care. Implement Sci 2010, 5:38.

35. Rycroft-Malone J, Wilkinson J, Burton C, Andrews G, Ariss S, Baker R, Dopson S, Graham I, Harvey G, Martin G, McCormack B, Staniszewska S, Thompson C: Implementing health research through academic and clinical partnerships: a realistic evaluation of the Collaborations for Leadership in Applied Health Research and Care (CLAHRC). Implement Sci 2011, 6:74

36. Seers K, Cox K, Crichton N, Edwards R, Eldh A, Estabrooks C, Harvey G, Hawkes C, Kitson A, Linck P, McCarthy G, McCormack B, Mockford C, Rycroft-Malone J, Titchen A, Wallin L: FIRE (facilitating implementation of research evidence): a study protocol. Implement Sci 2012, 7:25.

37. Marchal B, van Belle S, van Olmen J, Hoerée T, Kegels G: Is realist evaluation keeping its promise? A review of published empirical studies in the field of health systems research. Evaluation 2012, 18:192-212.

38. Pawson R, Tilley N: Realistic Evaluation. London: SAGE Publications; 1997.

39. Blackwood B, O'Halloran P, Porter S: On the problems of mixing RCTs with qualitative research: the case of the MRC framework for the evaluation of complex healthcare interventions. J Res Nurs 2010, 15:511-521.

40. Koshy P, Mackenzie M, Leslie W, Lean M, Hankey C: Eating the elephant whole or in slices: views of participants in a smoking cessation intervention trial on multiple behaviour changes as sequential or concurrent tasks. BMC Public Health 2012, 12:500.

41. Mackenzie M, Koshy P, Leslie W, Lean M, Hankey C: Getting beyond outcomes: a realist approach to help understand the impact of a nutritional intervention during smoking cessation. Eur J Clin Nutr 2009, 63:1136-1142.

42. Byng R, Norman I, Redfern S: Using realistic evaluation to evaluate a practice-level intervention to improve primary healthcare for patients with long-term mental illness. Evaluation 2005, 11:69-93.

43. Byng R, Norman I, Redfern S, Jones R: Exposing the key functions of a complex intervention for shared care in mental health: case study of a process evaluation. BMC Health Serv Res 2008, 8:274

44. Pawson R: Theorizing the Interview. Br J Sociol 1996, 47:295-314.

45. Pope C, Ziebland S, Mays N: Analysing qualitative data. In Qualitative Research in Health Care. Edited by Pope C, Mays N. Oxford: Blackwell Publishing/BMJ Books; 2006:63-81.

46. Pawson R, Greenhalgh T, Harvey G, Walshe K: Realist review - a new method of systematic review designed for complex policy interventions. J Health Serv Res Policy 2005, 10:21-34

47. Undre S, Sevdalis N, Healey A, Darzi A, Vincent C: Observational teamwork assessment for surgery (OTAS): refinement and application in urological surgery. World J Surg 2007, 31:1373-1381.

48. Catchpole K, Wiegmann D: Understanding safety and performance in the cardiac operating room: from 'sharp end' to 'blunt end'. BMJ Qual Saf 2012, 21:807-809.

49. Catchpole KR: Task, team and technology integration in the paediatric cardiac operating room. Prog Pediatr Cardiol 2011, 32:85-88.

50. Catchpole KR, Giddings AEB, de Leval MR, Peek GJ, Godden PJ, Utley M, Gallivan S, Hirst G, Dale T: Identification of systems failures in successful paediatric cardiac surgery. Ergonomics 2006, 49:567-588.

51. Schraagen JM, Schouten T, Smit M, Haas F, van der Beek D, van de Ven J, Barach P: Assessing and improving teamwork in cardiac surgery. Qual Saf Health Care 2010, 19:1-6.

52. Greenhalgh T, Swinglehurst D: Studying technology use as social practice: the untapped potential of ethnography. BMC Med 2011, 9:45.

53. Hammersley M, Atkinson P: Ethnography: Principles in Practice. London: Routledge; 1995

54. Emerson R, Fretz R, Shaw L: Writing Ethnographic Fieldnotes. Chicago: University of Chicago Press; 1995

55. McDonald S: Studying actions in context: a qualitative shadowing method for organizational research. Qual Res 2005, 5:455-473.

56. Heath C, Hindmarsh J, Luff P: Video in Qualitative Research: Analysing Social Interaction in Everyday Life. London: Sage; 2010

57. Garfinkel H: Studies in Ethnomethodology. Cambridge: Polity Press; 1967.

58. Sacks H: Lectures on Conversation: Volumes / \& II. Oxford: Blackwell; 1995.

59. Campbell NC, Murray E, Darbyshire J, Emery J, Farmer A, Griffiths F, Guthrie $\mathrm{B}$, Lester $\mathrm{H}$, Wilson $\mathrm{P}$, Kinmonth AL: Designing and evaluating complex interventions to improve health care. Br Med J 2007, 334:455-459.

60. Craig P, Dieppe P, Macintyre S, Michie S, Nazareth I, Petticrew M: Developing and evaluating complex interventions: the new Medical Research Council guidance. BMJ 2008, 337:a1655.
61. Cook JA, McCulloch P, Blazeby JM, Beard DJ, Marinac-Dabic D, Sedrakyan A IDEAL framework for surgical innovation 3: randomised controlled trials in the assessment stage and evaluations in the long term study stage. BMJ 2013, 346:f2820.

62. McCulloch P, Cook JA, Altman DG, Heneghan C, Diener MK: IDEAL framework for surgical innovation 1: the idea and development stages. BMJ 2013, 346:f3012

63. Eveleigh $\mathrm{MO}$, Blencowe NS, Mills N, Blazeby JM: Understanding the complexity of surgical procedures in RCTs: a pilot study to test the application of the MRC framework for evaluating complex healthcare interventions in the operating theatre. Trials 2011, 12:A148.

64. Wolff N: Randomised trials of socially complex interventions: promise or peril? J Health Serv Res Policy 2001, 6:123-126.

65. Lindsay B: Randomized controlled trials of socially complex nursing interventions: creating bias and unreliability? J Adv Nurs 2004, 45:84-94.

66. Bonell C, Fletcher A, Morton M, Lorenc T, Moore L: Realist randomised controlled trials: A new approach to evaluating complex public health interventions. Soc Sci Med 2012, 75:2299-2306.

67. Bonell C, Fletcher A, Morton M, Lorenc T, Moore L: Methods don't make assumptions, researchers do: a response to Marchal et al. Soc Sci Med 2013, 94:81-82.

68. Marchal B, Westhorp G, Wong G, Van Belle S, Greenhalgh T, Kegels G, Pawson R: Realist RCTs of complex interventions - an oxymoron. Soc SCi Med 2013, 94:124-128.

69. Blamey A, Mackenzie M: Theories of change and realistic evaluation: peas in a pod or apples and oranges? Evaluation 2007, 13:439-455.

doi:10.1186/1748-5908-9-52

Cite this article as: Randell et al.: Integration of robotic surgery into routine practice and impacts on communication, collaboration, and decision making: a realist process evaluation protocol. Implementation science 2014 9:52

\section{Submit your next manuscript to BioMed Central and take full advantage of:}

- Convenient online submission

- Thorough peer review

- No space constraints or color figure charges

- Immediate publication on acceptance

- Inclusion in PubMed, CAS, Scopus and Google Scholar

- Research which is freely available for redistribution 